Ann. Biol. anim. Bioch. Biophys., 1977, 17 (5 B), 765-774.

\title{
Transformation de la graisse brune en graisse blanche. Arguments morphologiques et fonctionnels (comportement vis-à-vis des corticostéroïdes au cours d'une agression)
}

\author{
par M. THONNERIEUX, D. GUERRIER, Michèle VIGIER, Hélène PELLET
}

Laboratoire d'Histologie-Embryologie,

U.E.R. Médicale Grange-Blanche,

8, avenue Rockefeller, 69373 Lyon Cedex 2.

Summary. Morphological and functional reasons for the transformation of brown fat into white fat : Correlation with corticosteroid content during stress.

The spontaneous morphologic evolution of brown adipose tissue differs according to animal species ; in some species (cat, dog, guinea-pig, monkey) brown fat is precociously replaced by white fat, while in other species (golden hamster, hedgehog, rat) typical brown adipose tissue is observed during all life.

White adipose cells seem to be the result of a progressive evolution of brown adipocytes, since ultrastructural intermediary aspects between brown and white cells are described. In experimental stress conditions (ether exposure, histamine injection) lipidic vacuoles in brown adipose tissue disappear, except in some unilocular or paucilocular cells which do not respond to the stimulus.

In the rat, acute stress results in higher corticosterone levels in brown fat than in white fat. In the histamine-treated guinea-pig, the correlation between plasma cortisol concentrations and cortisol content in the brown fat decreases during the first days of life. It is assumed that the functional alterations are related to the progressive transformation of brown fat into white fat.

\section{Introduction.}

Bien que posséda'nt une topographie, une morphologie ef un métabolisme propres, la graisse brune est considérée par certains auteurs (Sidman, 1956 ; Johansson, 1959 ; Simon, 1962) comme une forme embryonnaire de graisse blanche. Mais la majorité des auteurs (Napolitano et Fawcett, 1958 ; Hull, 1966, Smalley ef Smalley, 1967 ; Daniel et Derry, 1969) pensent au contraire qu'il s'agit de deux tissus différents. Dans cette hypothèse, il est vraisemblable que la graisse brune se transforme en graisse blanche par un phénomène de dégénérescence. Ce travail compare dans plusieurs espèces animales l'évolution morphologique spontanée du tissu adipeux brun en fonction de l'âge. D'autre part, l'effet d'une agression aiguë sur la morphologie ef 
sur la teneur en corticostéroïdes de la graisse brune est étudié chez le rat adulte, dont le tissu adipeux brun demeure bien développé, et chez le cobaye à la période postnatale, où la graisse brune est rapidement remplacée par la graisse blanche.

\section{Matériel et méthodes.}

Animaux. L'évolution morphologique spontanée est observée sur des espèces non hibernantes : chat et chien, cobaye (Hartley), lapin (Néozélandais), rał (SpragueDawley), singe (Macacus Irus, Cynocéphale, Vervet) et chez des espèces hibernantes dans les conditions naturelles : graphiure (Graphiurus murinus), hamster doré (Mesocricetus auratus), hérisson (Erinaceus europaeus) et spermophile (Citellus variegatus grammurus). Les singes, les graphiures et les spermophiles sont des adultes; pour les autres espèces, nous étudions des fœius au voisinage du terme, et des animaux de la naissance à l'âge adulte.

Prélèvement des échantillons. Les prélèvements de graisse brune sont effectués dans la région scapulo dorsale et accessoirement périrénale (chez le chien ef le singe). Seuls sont utilisés pour le dosage des corticostéroïdes les fragments ayant l'aspect de graisse brune, débarrassés par une dissection soigneuse de tout élément fibreux et des parties de couleur blanche. Un contrôle en microscopie photonique est effectué de façon systématique. Chez le rat, on recueille de 150 à $300 \mathrm{mg}$ de graisse brune par animal, ce qui impose l'utilisation de 3 ou 4 animaux pour un dosage. La graisse blanche abdominale est également groupée, 2 à $3 \mathrm{~g}$ étant nécessaires par essai. Chez le cobaye, au cours de la période étudiée (de la naissance au $12 \mathrm{e}$ jour), l'abondance de la graisse brune ( 2 à $4 \mathrm{~g}$ par animal) permet d'effectuer des dosages individuels de cortisol.

\section{PLANCHE I}

FIG. 1. - Graisse brune inferscapulaire de foetus de hérisson proche du terme. Noter la charge lipidique encore très modérée, la coexistence d'adipocytes bruns multiloculaires et de cellules du type précurseur, uniloculaires. $G \times 500$, immersion.

FIG. 2. - Graisse brune inferscapulaire de hamster doré nouveou-né. Le tissu est encore très peu organisé, avec de vastes plages acellulaires. On ne trouve pas d'adipocytes bruns, mais seulement des précurseurs uniloculaires et quelques amas de cellules dépourvues de lipides, décrites par Smalley comme des cellules de graisse brune immatures. $G \times 256$.

FIG. 3. - Graisse brune interscapulaire de chien âgé de $30 \mathrm{~h}$. Au faible grossissement, le tissu paraît presque exclusivement constitué de cellules encore non chargées de lipides. $G \times 78,5$.

FIG. 4. - Même préparation que figure 3 , à un plus fort grossissement. On remarque la présence de quelques vacuoles lipidiques intracellulaires, la majorité des cellules sont de type plurivacuolaire. $\mathrm{G} \times 312$.

FIG. 5. - Graisse brune périsurrénale de chat âgé de 30 jours. La plupart des cellules sont des adipocytes bruns typiques multiloculaires. Quelques-uns, encore multiloculaires, ont des vacuoles volumineuses plus ou moins confluentes. $\mathrm{G} \times 312$.

FIG. 6. - Graisse brune interscapulaire de hamster doré adulte. Adipocyłes bruns multiloculaires. Quelques cellules à très grande vacuole paraissant unique. $G \times 200$. 
Agression provoquée. Chez des rats des deux sexes, âgés de 4 à 6 semaines, l'agression aiguë est réalisée par exposition aux vapeurs d'éther en atmosphère confinée pendant $10 \mathrm{mn}$ (l'animal étant remis quelques instants à l'air libre en cas de troubles respiratoires). Aux temps zéro, $1 \mathrm{~h}$ ou $3 \mathrm{~h}$ après l'exposition, les rats sont décapités et les tissus immédiatement prélevés. Des rats témoins sont sacrifiés moins d'une minute après l'ouverture de la cage. Chez des cobayes des deux sexes, aux jours 2 , 5, 8, l'agression aiguë est provoquée par une injection sous-cutanée de chlorhydrate d'histamine à la dose de $5 \mathrm{mg}$ par kilo de poids corporel, dans une solution de chlorure de sodium à 9 p. 1000 , et les animaux sont sacrifiés par ponction cardiaque sous anesthésie au nembutal.

Microscopie photonique. Après fixation au liquide de Bouin Hollande et inclusion en paraffine, les coupes de $5 \mu$ sont colorées par le trichrome de Masson.

Microscopie électronique. La fixation est faite au tétroxyde d'osmium en tampon cacodylate, l'inclusion dans l'araldite selon Richardson et al. (1960) ef le contraste selon Reynolds (1963). L'examen est fait au microscope électronique JEM 7 à $80 \mathrm{KW}$. Des coupes semi-fines sont colorées selon la méthode de Richardson et al. (1960).

Dosage des corticostéroïdes. Les tissus sont homogénéisés dans une solution éthanolique à 30 p. 100 . Le cortisol ef la corticostérone sont dosés dans le plasma ef les homogénats de surrénale, de graisse brune et de graisse blanche, par induction de fluorescence, après purification par l'iso-octane et l'éther de pétrole, extraction par le dichlorométhane ef séparation sur colonne de gel de silice, selon la méthode

\section{PLANCHE II}

FIG. 7. - Graisse brune inferscapulaire de cobaye âgé de 2 jours. Cellules multiloculaires à volumineuses vacuoles confluentes et à cyłoplasme extrêmement réduit. $G \times 500$, immersion.

FIG. 8. - Graisse brune interscopulaire de chat âgé de $48 \mathrm{~h}$. Zone de transition : à gauche graisse brune en transformation, quelques cellules multiloculaires, des cellules à grande vacuole unique entourée d'un cytoplasme granuleux ; à droite aspect d'adipocytes blancs, le cytoplasme est réduit au liséré entourant la vacuole. $\mathrm{G} \times 312$.

FIG. 9. - Graisse brune interscopulaire de chien âgé de 3 semaines. A gauche, fragment d'adipocyte blanc: liséré cytoplasmique très fin limitant une vacuole lipidique. A droite, fragment d'adipocyte brun : plusieurs vacuoles lipidiques ef nombreuses mitochondries dans le cytoplasme. $\mathrm{G} \times 8000$.

FIG. 10. - Graisse brune interscapulaire de rat stressé. Disparition des lipides dans la majorité des celJules réalisant un aspect d'hépatisation. Quelques cellules univacuolaires situées le long des axes conjonctifs contrastent par la persistance de leur charge lipidique. $\mathbf{G} \times 80$.

FIG. 11. - Même préparation que fig. 9. Fragment d'adipocyte brun en cours de transformation : le noyau est aplati et périphérique comme dans un adipocyte blanc, le cytoplasme est refoulé par contenu lipidique, mais les mitochondries restent nombreuses. G Y. 8000 .

FIG. 12. - Graisse brune de rat adulte. Coupe semi-fine, coexistence d'adipocytes multiloculaires typiques (les points denses sont les mitochondries) et de cellules uni -ou paucivacuolaires distendues par le contenu lipidique mais dont le cytoplasme contient encore des mitochondries. $G$ y. 500, immersion. 


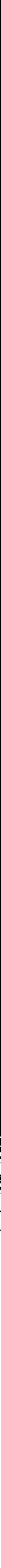


mise au point au laboratoire (Guerrier ef al., 1975, 1976). Les résultats sont exprimés en $\mu \mathrm{g} / 100 \mathrm{ml}$ pour le plasma ef en $\mu \mathrm{g} / 100 \mathrm{~g}$ pour les tissus. Les moyennes sont calculées avec l'intervalle de confiance à $P=0,05$.

\section{Résultats.}

A. Données morphologiques.

1. Evolution spontanée de la graisse brune. Un remplacement spontané de la graisse brune par la graisse blanche est observé selon une chronologie dépendant des espèces.

A la naissance, la graisse brune des lobes interscapulaires est encore de type embryonnaire chez le chien (pl. l, fig. 3 et 4), le hamster doré (pl. l, fig. 2), le hérisson, alors que la transformation en graisse blanche est déjà en cours chez le cobaye et le lapin.

Au $2^{e}$ jour, la graisse brune interscapulaire du chien reste embryonnaire, alors que les adipocyłes bruns du chat, du cobaye, du lapin, sont très chargés en lipides, évoluant vers l'aspect de la graisse blanche. Des images du même type sont trouvées chez le chien âgé de 3 semaines (pl. Il, fig. 11). Chez le graphiure et le spermophile adultes, les lobes interscapulaires sont réduits à quelques éléments fibro-conjonctifs dépourvus d'adipocytes bruns, mais les autres localisations cervico-dorsales contiennent de la graisse brune typique. En revanche, la transformation en graisse blanche des lobes interscapulaires n'a jamais lieu chez le rat ef le hamster doré (pl. l, fig. 6), même âgés.

Cette évolution comporte des images intermédiaires entre adipocyle brun et blanc : en microscopie photonique (pl. II, fig. 7 et 8 ), et sur coupes semi-fines (pl. II, fig. 12), une augmentation du volume et une confluence des vacuoles lipidiques, refoulant à la périphérie le cytoplasme et le noyau qui s'aplatit, le dernier caractère distinctif de l'adipocyte brun étant l'aspect granuleux du cytoplasme ; en microscopie électronique ( $\mathrm{pl}$. Il, fig. 11), des cellules où, dans l'anneau cyłoplasmique très fin cernant une énorme vacuole lipidique, les mitochondries sont plus nombreuses que dans un adipocyte blanc.

2. Aspect de la graisse brune après le stress. L'agression par l'éther ou l'histamine produit une déplétion lipidique brutale de la graisse brune (pl. II, fig. 10). Les vacuoles ne sont généralement plus visibles en microscopie photonique, réalisant une véritable hépatisation du tissu, mais la microscopie électronique montre la persistance de petites vacuoles. On observe cependant chez le rat (pl. Il, fig. 10) quelques cellules, toujours univacuolaires, du moins en microscopie photonique, qui ne se vident pas de leurs lipides. Ces cellules sont, soit isolées en plein parenchyme, soit groupées le long des axes conjonctifs ou en bordure des lobes. Chez le cobaye nouveau-né, ces cellules ne répondant pas à l'agression par une déplétion lipidique sont beaucoup plus nombreuses, formant de larges plages.

\section{B. Teneur en corticostéroïdes.}

1. Graisse brune du rat adulte. La teneur en corticostérone du plasma, des surrénales et des deux tissus adipeux des rats adultes témoins et des rats exposés aux vapeurs 
d'éther est représentée sur la figure 13. L'agression provoque une augmentation très rapide de la concentration en corticostérone dans le plasma et les tissus étudiés. Cette élévation se maintient $1 \mathrm{~h}$ plus tard, mais au temps $3 \mathrm{~h}$ après le stress, on constate une tendance au retour aux valeurs observées chez les rats témoins. D'autre part, l'élévation dans la graisse brune est beaucoup plus forte que dans la graisse blanche.

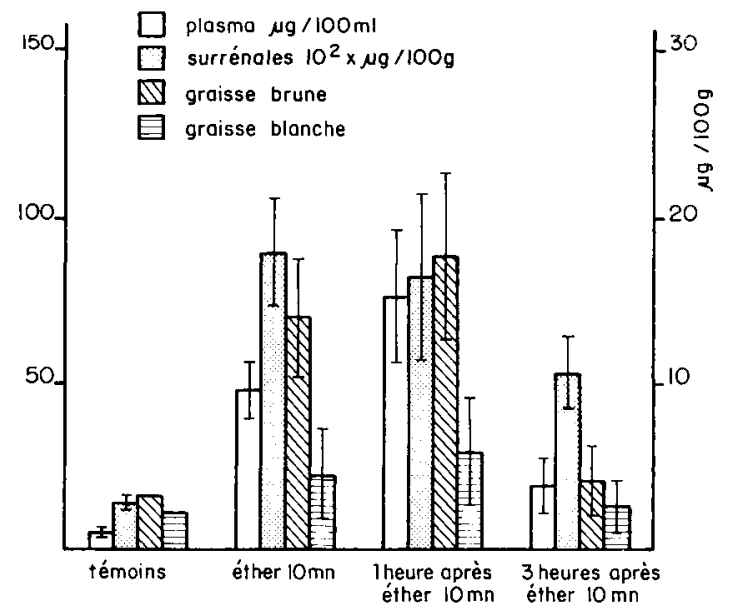

FIG. 13. - Concentrations en corticostérone dans le plasma, ordonnées de gauche, les surrénales, la graisse brune et la graisse blanche, ordonnées de droite, chez le rat exposé oux vapeurs d'éther. Les traits verticaux représentent l'intervalle de confiance à $P=0,05 . n=9$ à 11 .

2. Graisse brune du cobaye nouveau-né. La teneur en cortisol du plasma et de la graisse brune du cobaye entre la naissance et le $12^{\mathrm{e}}$ jour est représentée sur les figures 14 et 15. La concentration de cortisol plasmatique, élevée à la naissance,

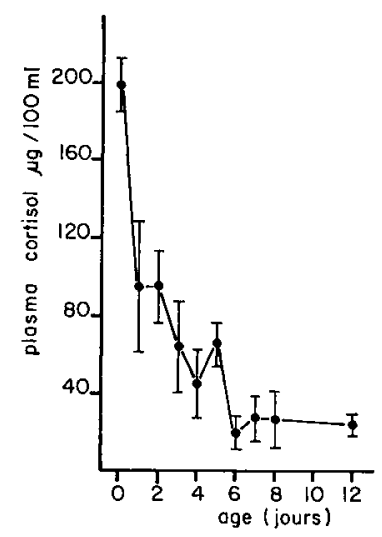

FIG. 14. - Evolution de la concentration en cortisol plasmatique chez le cobaye âgé de 0 à 12 jours. Les traits verticaux représentent l'intervalle de confiance à $P=0,05 . n=4$ à 9 .

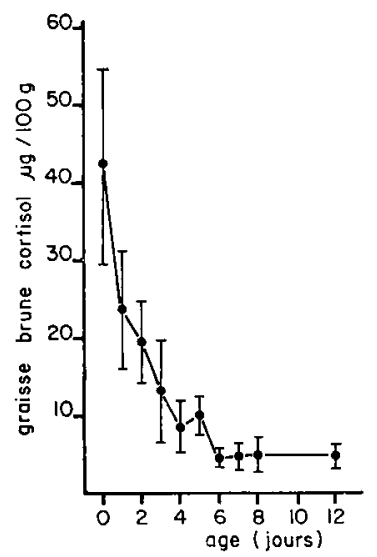

FIG. 15. - Evolution de la concentration en cortisol dans la graisse brune chez le cobaye âgé de 0 à 12 jours. Les traits verticaux représentent l'intervalle de confiance $\dot{a} \mathrm{P}=0,05$. $n=4$ à 10. 
s'abaisse progressivement jusqu'au 6e jour, puis se stabilise. La concentration de cortisol dans la graisse brune suit une courbe analogue. Le coefficient de corrélation, calculé pour une régression linéaire de type $y=a x+b$, est supérieur à 0,89 , démontrant la proportionnalité entre les concentrations de cortisol dans le plasma et le tissu adipeux brun (fig. 16). Cependant, cette corrélation diminue chez les animaux soumis à une agression par l'histamine (voir tabl. 1) : la quantité de cortisol trouvée dans le tissu adipeux brun n'est plus proportionnelle à celle du plasma, et ce phénomène s'accentue avec l'âge.

\section{TABLEAU 1}

Corrélation entre les concentrations en cortisol dans le plasma et dans la graisse brune chez le Cobaye traité par l'hisłamine

\begin{tabular}{cc}
\hline âge (jours) & $r$ \\
& \\
2 & 0,505 \\
5 & 0,450 \\
8 & 0,003 \\
\hline
\end{tabular}

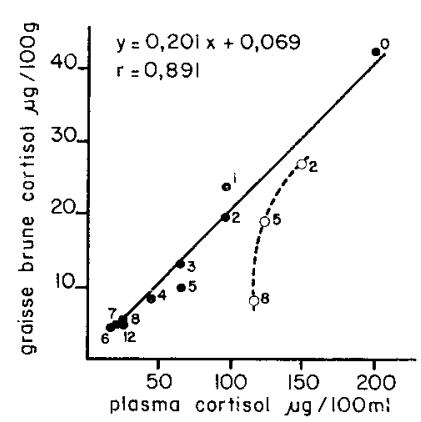

FIG. 16. - Evolution des concentrations en cortisol dans la graisse brune en fonction des concentrations en cortisol plasmatique chez le cobaye (animaux témoins, $O$ animaux traités par l'histamine). Les chiffres indiquent l'âge en jours.

\section{Discussion.}

L'observation d'aspects intermédiaires dans diverses espèces animales, comme l'absence d'autres précurseurs éventuels, suggèrent fortement une transformation progressive des adipocytes bruns en adipocytes blancs. Allant de pair avec cette évolution morphologique, nous observons des modifications fonctionnelles : l'agression par l'éther ou l'histamine met en évidence dans le tissu adipeux brun des cellules déjà transformées ou en cours de transformation en adipocytes blancs, qui ne se comportent plus comme des adipocytes bruns, et restent chargées de lipides. Chez le cobaye nouveau-né, l'altération fonctionnelle des adipocytes bruns se traduit par leur comportement différent vis-à-vis des corticostéroïdes circulants. 
L'agression aiguë par l'éther ou l'histamine déclenche une libération massive d'hormone corticotrope et de catécholamines. La première élève le taux des corticoïdes plasmatiques, la noradrénaline produit un déplétion lipidique brutale de la graisse brune (Dirschmid, 1972). Son action sur ce point l'emporte sur celle de l'ACTH (Baker ef al. 1950 ; Pellet et al., 1975) et des hormones corticoïdes (Aronson et al., 1954 ; Pellet ef al., 1975) qui, en administration chronique, augmentent au contraire la charge lipidique des adipocytes bruns.

Chez le rat adulte stressé, dont le tissu adipeux brun multiloculaire typique répond à l'agression par une déplétion lipidique intense et brutale, l'élévation du taux de corticostérone plasmatique s'accompagne d'une forte élévation de ce stéroìde dans la graisse brune.

Au contraire, chez le cobaye nouveau-né stressé, l'élévation de cortisol dans la graisse brune en réponse à l'agression est d'autant moins marquée qu'on s'éloigne de la naissance, ef durant cette période, le tissu adipeux brun contient une proportion croissante d'adipocytes univacuolaires restant chargés en lipides au cours du stress.

\section{Conclusion.}

Les observations morphologiques confirment l'hypothèse d'une transformation des adipocyles bruns en adipocytes blancs. Cette transformation a lieu dès les premiers jours de la vie pour certaines espèces animales, elle n'est jamais observée pour d'autres. Lorsque la graisse brune a une morphologie multiloculaire typique, elle capte plus de corticostéroïdes endogènes que la graisse blanche.

L'absence de déplétion lipidique et la faible captation de corticostéroïdes en réponse au stress peuvent traduirent la transformation du tissu adipeux brun en tissu adipeux blanc.

Réunion Groupe Développement INRA/Productions animales Thivervol-Grignon, 14-15 ovril 1976.

\section{Références}

ARONSON S. M., TEODORU C. V., ADLER M., SCHWARTZMAN G., 1954. Influence of cortisone upon brown fat of hamsters and mice. Proc. Soc. exp. Biol. Med., 85, 214-218.

BAKER B. L., INGLE D. J., LI C. H., 1950. Increase in glyceride content of brown fat by treatment with adrenocorticotrophin. Proc. Soc. exp. Biol. Med., 73, 337-339.

DANIEL H., DERRY M. D., 1969. Criteria for differentiation of brown and white fat in the rat. J. Physiol. Pharmacol., 47, 941-945.

DIRSCHMID K., 1972. Die Pharmacodynamische Beeinflussung des fierischen braunen Fettgewebes. Exp. Path., 6, 346-349.

GUERRIER D., THONNERIEUX M., PELLET H., 1975. Dosage du cortisol et de la corticostérone dans le plasma ef les surrénales de hamster doré (Mesocricetus auratus). C. R. Acad. Sci. Paris, Série $D, 280,2781-2784$.

GUERRIER D., THONNERIEUX M., VIGIER M., PELLET H., 1976. Influence des conditions de prélèvement sur la concentration en corticostérone dans la graisse brune de raf. C. R. Acad. Sci. Paris, Série $D, 282,759-762$.

HULL D., 1966. The structure and function of brown adipose tissue. Brit. med. Bull., 22, 92-96.

JOHANSSON B. W., 1959. Brown fat : a review. Metabolism, 8, 221-240. 
NAPOLITANO L., FAWCETT D. W., 1958. The fine structure of brown adipose tissue in the newborn mouse and rat. J. biophys. biochem. Cytol., 4, 685-692.

PELLET H., GUERRIER D., THONNERIEUX M., VIGIER M., 1975. Influence de l'axe hypophysosurrénalien sur le tissu adipeux brun chez le hamster doré. Bull. Ass. Anat., 59, 479-488.

REYNOLDS E. S., 1963. The use of lead citrate at high $\mathrm{pH}$ as an electron opaque stain in electron microscopy. J. Cell Biol., 17, 208-212.

RICHARDSON H. C., JARETT L., FINKE E. H., 1960. Embedding in epoxy for electron microscopy. Stain Technol., 35, 313-323.

SIDMAN R. L., 1956. Histogenesis of brown adipose tissue in vivo and in organ culture. Anat. Rec., 124, 581-601.

SIMON G., 1962. Genèse et structure du tissu adipeux chez l'homme. Acta Anat., 48, 232-241.

SMALLEY R. L., SMALLEY K. N., 1967. Brown and white fats : development in the hamster. Science, 157, 1449-1451. 